\title{
SPINDLE CELL RHABDOMYOSARCOMA MASQUERADING AS PSEUDO-TUMOUR OF THE ORBIT
}

\author{
Anitha Chakravarthy', Teerthanath Srinivas ${ }^{2}$ \\ 1 Junior Resident, Department of Pathology, KS Hegde Medical Academy, Deralakatte, Mangalore. \\ 2Professor, Department of Pathology, KS Hegde Medical Academy, Deralakatte, Mangalore.
}

\section{ABSTRACT}

Paediatric solid neoplasms are a global burden causing highest incidence of mortality in children. Among the soft tissue tumours in children, Rhabdomyosarcoma is the most common. They possess variety of histologies that differ among various age groups. Here, we discuss a case of a 3-year-old child diagnosed with embryonal rhabdomyosarcoma, which initially mimicked an orbital pseudotumour. Histopathology and Immunohistochemistry confirmed the diagnosis. This case report has uncommon clinical and radiological presentation of rhabdomyosarcoma.

\section{KEYWORDS}

Embryonal Rhabdomyosarcoma, Spindle Cell Variant, Orbital Pseudo Tumour.

HOW TO CITE THIS ARTICLE: Chakravarthy A, Srinivas T. Spindle cell rhabdomyosarcoma masquerading as pseudo-tumour of the orbit. J. Evolution Med. Dent. Sci. 2016;5(23):1270-1272, DOI: 10.14260/jemds/2016/297

\section{INTRODUCTION}

Malignant solid tumours in childhood has seen a rise over the decades and is a major cause of mortality.[1] Tumours in paediatric age group are diverse and are a challenge to the diagnostic pathologist.[2] In this report we discuss a case of embryonal rhabdomyosarcoma, which was initially diagnosed as orbital pseudo-tumour.

\section{CASE REPORT}

A 3-year-old male child presented to the Ophthalmology Department with complaints of swelling of the right eye since one month. It was associated with fever, pain and purulent discharge. Local clinical examination revealed ptosis of the right eye with lid oedema and discharge causing stickiness of the eye lashes. A firm mass was palpable on the medial and inferior aspect of the orbit. Fundus examination showed a cup:disc ratio of 0.3 and retinal oedema in the macula. General physical examination was unremarkable. Systemic examination was normal. There was no evidence of focal neurological deficits. The Haematological and Biochemical parameters were normal. A clinical diagnosis of inflammatory orbital lesion was made.

Computerised Tomography scan of the orbit showed diffuse thickening of the medial rectus muscle, minimal thickening of the inferior rectus muscle on the right side with mass effect on the globe, mild proptosis and periorbital soft tissue thickening indicated inflammatory changes suggesting orbital pseudo-tumour (Figure 1). Magnetic Resonance Imaging of the orbit revealed a heterogeneous signal intensity mass lesion in the right orbital extraconal space measuring $31 \times 17 \times 26 \mathrm{~mm}$ abutting the medial and inferior rectus muscle and causing mild indentation on the medial wall of the globe with no intraconal extension. There was no extension into the paranasal sinuses, orbital apex or intracranium suggesting the possibility of Rhabdomyosarcoma (Figure 2).

Financial or Other, Competing Interest: None.

Submission 06-02-2016, Peer Review 03-03-2016,

Acceptance 09-03-2016, Published 21-03-2016.

Corresponding Author:

Dr. Anitha Chakravarthy,

Junior Resident, Department of Pathology,

KS Hegde Medical Academy, Deralakatte,

Mangalore-575018.

E-mail: anithachakravarthy@gmail.com

DOI: $10.14260 /$ jemds $/ 2016 / 297$
An incisional biopsy was performed and the tissue was sent for histopathology. To rule out the possibility of metastasis, chest X-ray and the Ultrasonography of the abdomen was done, which suggested normal study.

Histopathology revealed predominantly loosely arranged scattered spindle cells with eosinophilic cytoplasm. The spindle cells at places showed angulation with a few of them having pleomorphic nuclei. There were also a few scattered tadpole and caudate shaped cells with hyperchromatic nucleus, prominent nucleoli and eosinophilic cytoplasm, occasionally cells showed striation. There were small undifferentiated cells with hyperchromatic nucleus. Matrix appeared myxoid containing scattered eosinophils and lymphocytes. There were also areas of necrosis amidst the tumour cells (Figure 3, 4). Histological features were suggestive of spindle cell type of Embryonal Rhabdomyosarcoma. Immunohistochemistry for Myogenin and Myo-D1 were positive following which chemoradiation therapy and enucleation were planned. Due to the possibility of the child going into leukopenia, he was referred to a paediatric haematoncologist for further followup and management.

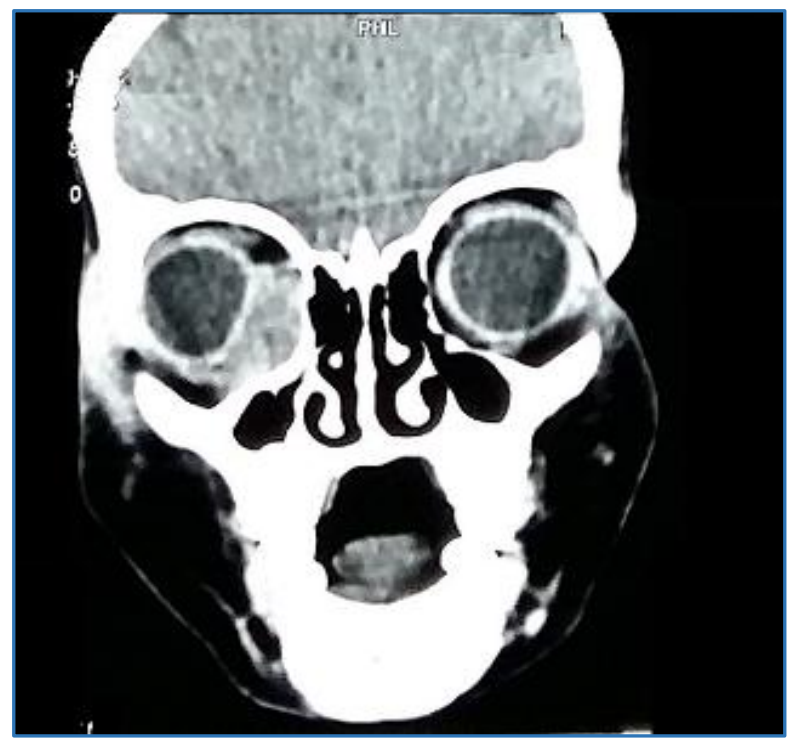

Fig. 1: Computerised Tomography scan showing diffuse thickening of medial rectus muscle and periorbital soft tissue in the right orbit 


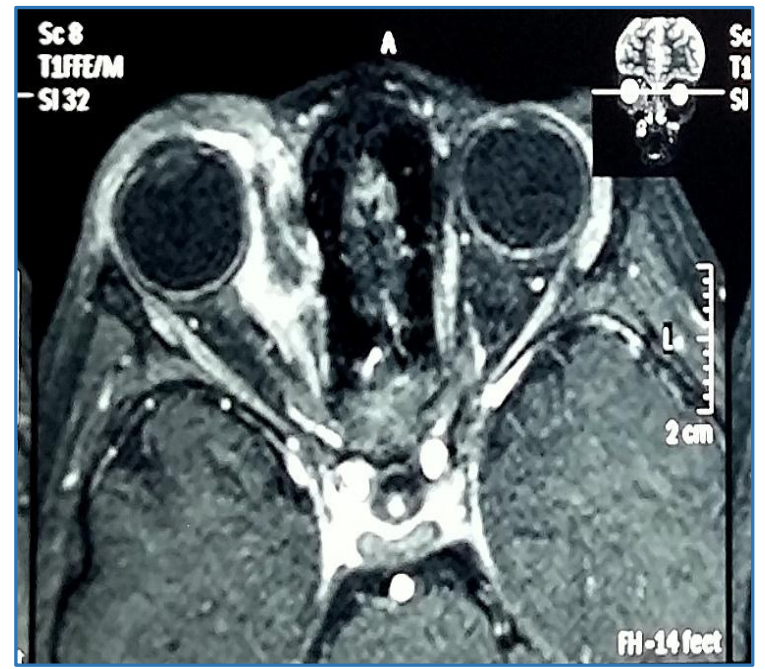

Fig. 2: Magnetic Resonance imaging showing heterogeneous signal intensity mass in the right medial and inferior conal space

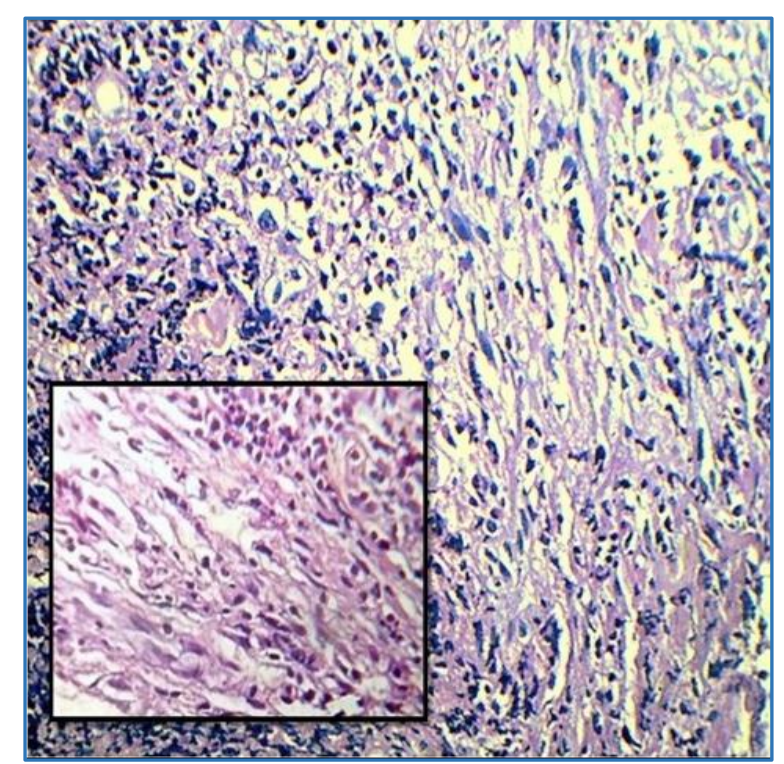

Fig. 3: Microscopy showing malignant spindle cells in abundant myxoid stroma with scattered neutrophils INSET: Showing pleomorphic spindle cells with hyperchromatic nucleus and few striations

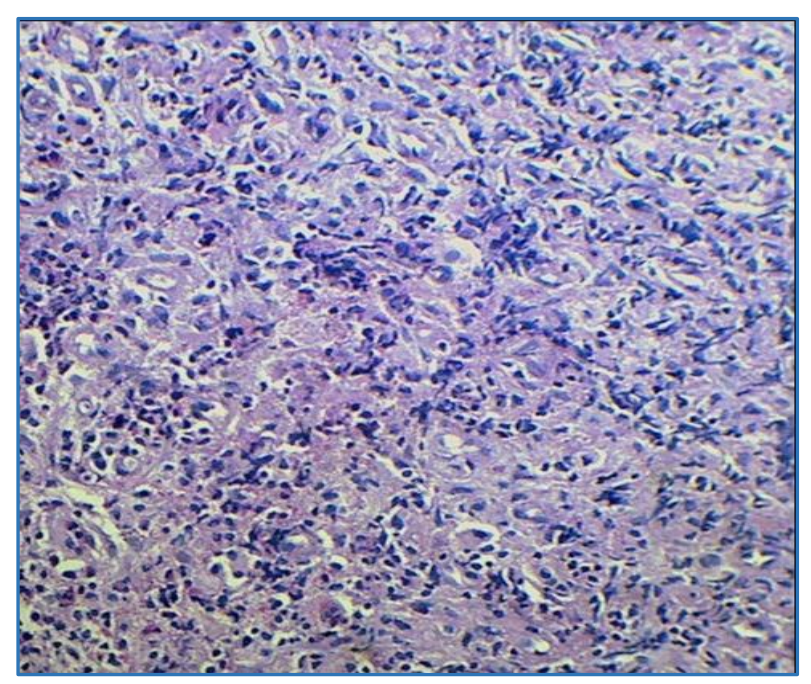

Fig. 4: Low power view showing cellular myxoid area containing malignant spindle cells, round cells and scattered lymphocytes and few eosinophils.

\section{DISCUSSION}

Paediatric solid malignant neoplasms cause significant mortality among children.[1] They constitute $2 \%$ of all the malignant tumours. The most commonly encountered malignancies in clinical practice include lymphoma, neuroblastoma, retinoblastoma, renal tumours, hepatic tumours, malignant bone tumours, soft tissue sarcoma and germ cell tumours. ${ }^{[2]}$ The incidence of soft tissue sarcomas in the United States of America is $10.7 \% .{ }^{[1]}$ and $5.49 \%$ in India. ${ }^{[3]}$

Rhabdomyosarcoma is a highly malignant soft tissue tumour.[4,5] It accounts to $5 \%$ of all the childhood cancers. ${ }^{[4]}$ The primary sites affected are the head and neck (40\%), genitourinary tract $(20 \%)$, extremities $(20 \%)$, trunk $(10 \%)$ and others (10\%). Amongst the paediatric age group, orbit is the most common site affected in the head and neck region accounting for $10 \%$ of all the rhabdomyosarcoma. The other ocular tissues affected include conjunctiva, eyelid and globe.[4] The most common clinical findings include proptosis, globe displacement, eyelid oedema and conjunctival congestion. ${ }^{[4,5]}$ These symptoms mimic various differential diagnosis which include inflammatory process, orbital cellulitis, metastatic neuroblastoma, chloroma, lymphangioma and ruptured dermoid cyst, which can be differentiated on history and orbital imaging.[6]

Rhabdomyosarcoma arise from pluripotent mesenchyme. [5] They often arise at sites in which striated muscle tissue is absent or scant. It was described as a distinct clinical entity by Arthur Purdy Stout.[6] Various classifications have been proposed by various agencies for rhabdomyosarcoma based on the morphology, clinical behaviour, differentiation and prognosis. Horn and Enterline in 1958 devised the first classification based on the pathological features into Embryonal, Botryoid, Alveolar and Pleomorphic subtypes. ${ }^{[6,7]}$

Embryonal Rhabdomyosarcoma is the most common variant, commonly seen in children less than 10 years.[7,8] Microscopically, uniform cellular proliferation of elongated spindle cells arrayed in fascicles and whorls are seen. The cells exhibit eccentric nuclei and bright cytoplasmic eosinophilia, occasionally exhibiting cross striations. [8] features of which is seen in our case report. It shows loss of heterozygosity for multiple loci at chromosome 11p15.5. Some studies have shown t (2; 8) (q35; q13) translocation, which involves PAX3, and $\mathrm{t}(4 ; 22)$ (q35;q12), which involved EWSR1.[7] Alveolar rhabdomyosarcoma comprise of nests of primitive cells that appear to float in the middle of alveolar spaces with a fibrovascular septa, which outline the cell clusters.[8] Pleomorphic rhabdomyosarcoma are high-grade sarcomas comprising of irregular, hyperchromatic nuclei and numerous mitoses.[8,9] Botryoid variant consists of cells varying from primitive small cells to cells with extensive myoblastic differentiation. The stroma is loosely cellular with a myxoid appearance including a hypocellular zone that separates the surface epithelium from the underlying cambium layer.[7] The rare variants of rhabdomyosarcoma include sclerosing variant and clear cell variant. ${ }^{[8]}$ In children, the primary lesions most commonly encountered are Ewing's sarcoma, neuroblastoma and lymphoma.[8]

Immunohistochemistry plays an important role in diagnosis and distinction of various types of rhabdomyosarcoma. The markers include antibodies against desmin (80-90\%), muscle-specific actin, Myo-D1 (70-90\%), 
myoglobin, vimentin and desmin. ${ }^{[5,7]}$ Vimentin and desmin are less specific as they are expressed in tumours with skeletal muscle differentiation. ${ }^{[5]}$

The staging for the management of orbital rhabdomyosarcoma can be done according to the Inter-group rhabdomyosarcoma study group (IRS), which is useful in terms of treatment stratification and prognosis prediction.[4,5] The clinical stage in this case was T2N0M0. Treatment includes surgery, irradiation and chemotherapy depending on the staging. Chemotherapy includes Vincristine for a period of 12 weeks, Actinomycin for a period of five to seven days and Cyclophosphamide for a period of two years. Radiotherapy should be started after the $9^{\text {th }}$ week and can be given upto 30 45Gy. The good prognosis is due to favourable location, histology and patient's age. Regular ocular examination has to be done every 3-4 months in the first year and then 4-6 months for several years and then yearly depending on the clinical findings and radiology.[4]

The followup of the patient in our case report could not be established due to the transfer of the patient to a higher centre with haematoncologist. The presentation of the case from the age of the child, symptoms manifested, clinical findings, radiological intervention and histopathological diagnosis coincided well with the facts published previously in literature.

\section{CONCLUSION}

Orbital rhabdomyosarcoma comprise of a wide clinical spectrum and can present diagnostic and therapeutic differences. Prompt treatment is indicated to improve the outcome and avoid blindness. Survival depends on the amount of residual tumour and the staging of the disease. A combination of chemotherapy, radiation therapy and surgery can increase the life expectancy of the child.

\section{REFERENCES}

1. Singh M, Harmon B, Friedman K, et al. Pediatric solid malignant neoplasms: a comparative analysis. Indian Journal of Pathology and Microbiology 2011;54(3):514-16.

2. Sharma S, Mishara K, Agarwal S, et al. Solid tumours of childhood. Indian J paediatr 2004;71:501-4.

3. Bhalodia JN, Patel MM. Profile of pediatric malignancy: a three year study. national Journal of Community Medicine 2011;2(1):24-27.

4. Shields C. Clinical spectrum of primary ophthalmic rhabdomyosarcoma. Ophthalmology 2001;108(12): 2284 92.

5. Jurdy L, Merks J, Pieters B, et al. Orbital rhabdomyosarcomas: a review. Saudi Journal of Ophthalmology 2013;27(3):167-75.

6. Das J, Tiwary B, Paul S, et al. Primary orbital rhabdomyosarcoma with skeletal muscle metastasis. Oman Journal of Ophthalmology 2010;3(2):91.

7. Hoda S. Enzinger and Weiss's soft tissue tumors, 6th Edition. Advances in anatomic pathology. 2014;21(3):216.

8. Carroll S, Nodit L. Spindle cell rhabdomyosarcoma: a brief diagnostic review and differential diagnosis. Archives of Pathology \& Laboratory Medicine 2013;137(8):1155- 58.

9. Parham DM, Ellison DA. Rhabdomyosarcomas in adults and children: an update. Arch Pathol Lab Med 2006;130:1454-65. 